\title{
Performance Tests of a Set of Gough-Reitzel Magnetic Variometers
}

\author{
F.E.M. Lilley, F.R. Burden, G.W. Boyd* and M.N. Sloane \\ Research School of Earth Sciences, \\ Australian National University, \\ Canberra, Australia
}

(Received July 15, 1974; Revised January 8, 1975)

\section{The Instruments}

Twenty-one magnetic variometers of the Gough-Reitzel (1967) pattern have been constructed at the Australian National University. The following minor departures from the description of the instrument as given in the 1967 paper are to be noted:

1.1. Following Professor Gough's own recent variometers (Serson, 1973), the instruments are shorter by some $50 \mathrm{~cm}$, with outside cases $138 \mathrm{~cm}$ in length. This reduction in length eases field operation, but introduces interactions between the $H$ and the $Z$ sensing magnets.

1.2. The lens in the instrument is achromatic. This enables cameras to be run without filters, and exposure times have been reduced to some $500 \mathrm{~ms}$. A major saving in battery power is achieved, and a $100 \mathrm{amp}-\mathrm{hr}$ car battery is sufficient for several $46 \mathrm{~m}$ (150 ft) lengths of film at the fastest recording speed of 6 exposures per minute (the exposures on the film being spaced $0.21 \mathrm{~mm}$ apart).

1.3. All timing is controlled electronically, with the basic circuit comprising a $1 \mathrm{MHz}$ quartz crystal oscillator and integrated circuits of low power consumption. The exposure and film advance interval may be set at $10 \mathrm{~s}, 30 \mathrm{~s}$ or $60 \mathrm{~s}$, giving a recording period of $3^{1} / 2$ weeks, 10 weeks or 21 weeks.

1.4. Timing marks are caused by a single large coil near the top of the instrument, through which current flows at the start of every hour for $30 \mathrm{~s}$, and at the start of every day for $600 \mathrm{~s}$.

1.5. Pulses to the camera to advance film are electrical rather than mechanical, as the solenoid and all subsequent gearing is now part of the camera itself.

* Now at the University of Melbourne, Victoria, Australia. 


\section{Field Tests}

All twenty-one instruments were tested simultaneously from July to October 1973 near the Toolangi observatory in south-east Australia, (geographic co-ords. $37^{\circ} 33^{\prime} \mathrm{S}, 145^{\circ} 28^{\prime} \mathrm{E}$ ). A plan of the installation is shown in Fig. 1. The data of this paper were obtained by reading peak-to-peak amplitudes for chosen events from the instrument records. Less than twenty-one points on a graph indicates that not all instruments were recording at the time of the event.

For a given event, the amplitude recorded by the $D$-sensor of an instrument will be proportional to the $D$-calibration deflection recorded by the same instrument. Thus if a graph is made for a particular $D$-event by plotting the $D$-amplitudes recorded by all instruments against their respective $D$-calibration deflections, a straight line should result, of slope proportional to the event amplitude. Three such graphs are given in Figs. 2a-c for response in the $D, H$ and $Z$ sensors respectively. Figure $2 c$ presents original data, and also corrections for $Z-H$ interactions, a point to be discussed in Section 3.4.

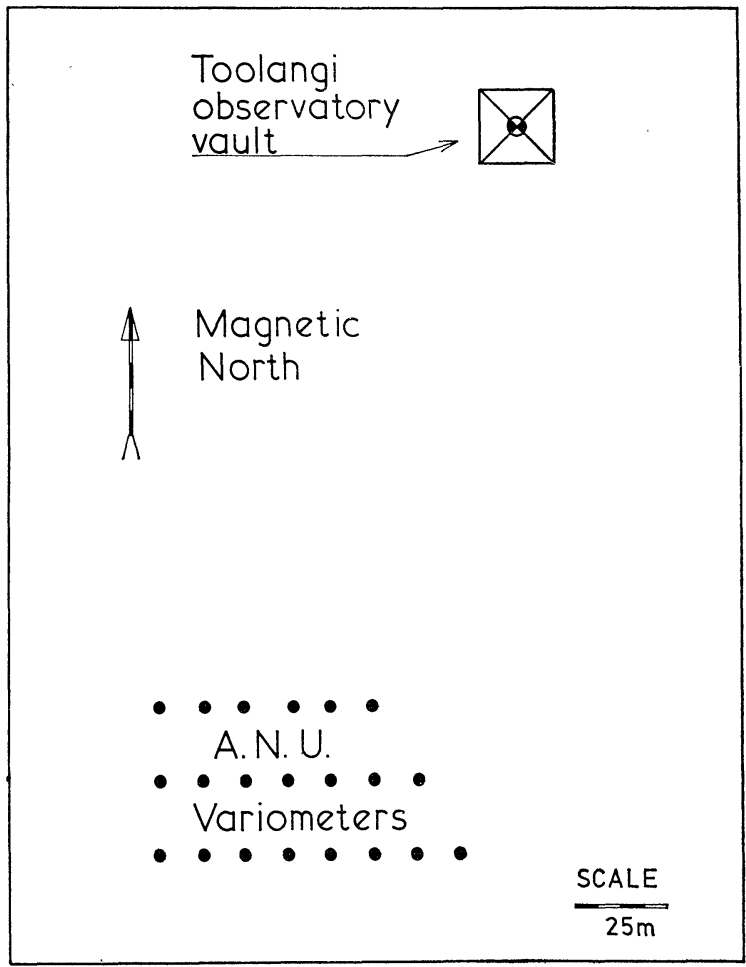

Fig. 1. Plan of installation near the Toolangi magnetic observatory, 1973. 


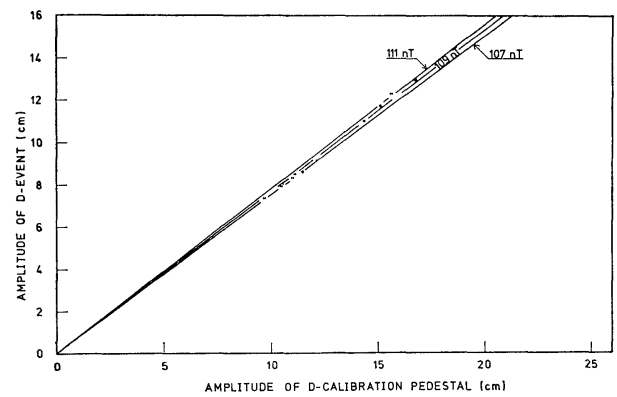

Fig. 2a. Data for an event in the $D$-component. The best line through the points has been given the value recorded by the observatory (109 nT), and from this have been determined the lines corresponding to events of amplitude $107 \mathrm{nT}$ and $111 \mathrm{nT}$, and a moment of 458 gauss$\mathrm{cm}^{3}$ for the variometer calibrating magnet, used subsequently.

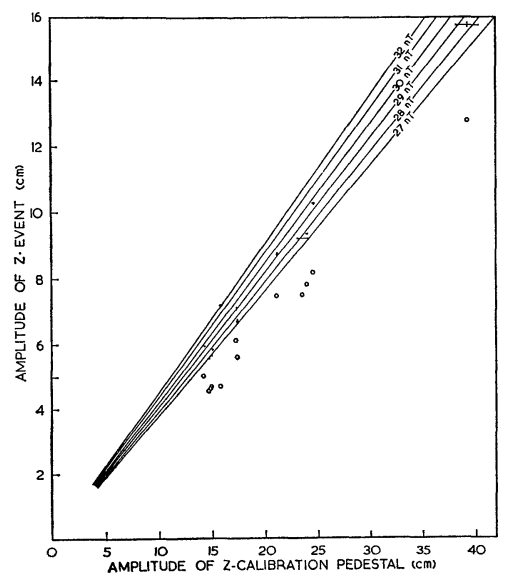

Fig. 2c. Data for an event in the $Z$-component. Amplitudes scaled from the instrument records are plotted as open circles. Each point has then been corrected (upwards) for $Z-H$ sensor interaction. The amplitude scaled off the observatory record was $29.5 \mathrm{nT}$ with standard deviation $0.7 \mathrm{nT}$.

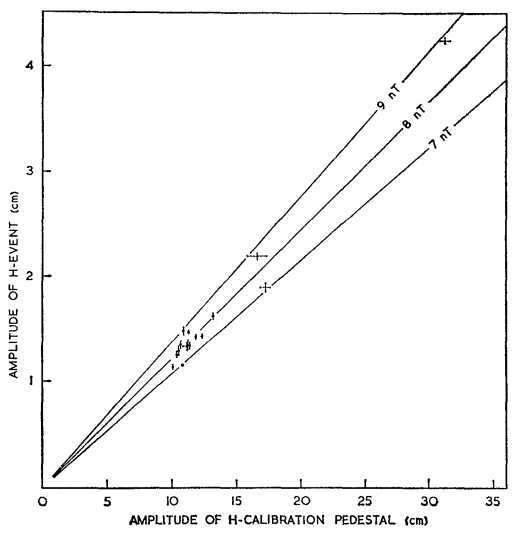

Fig. 2b. Data for an event in the $H$-component. The amplitude scaled off the observatory record was $7.1 \mathrm{nT}$ with standard deviation $0.3 \mathrm{nT}$.

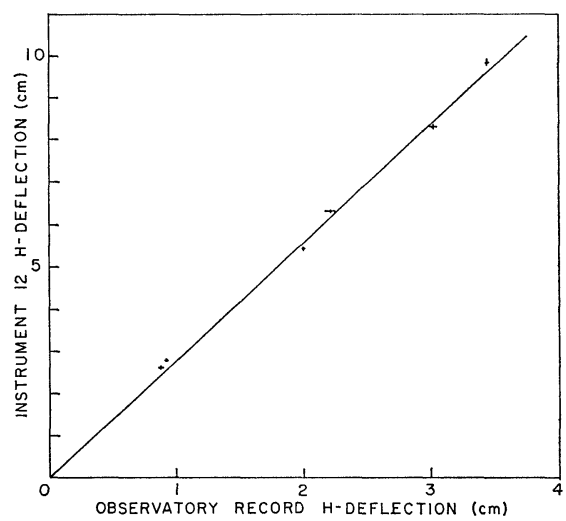

Fig. 2d. The $H$-sensor of instrument 12 checked for linearity against the $H$ component of the observatory, by plotting deflections of the former against deflections of the latter for events of different amplitudes. The line is theoretical, computed from calibrations of the two sensors. 


\section{Factors Limiting Variometer Accuracy}

In analysing the instrument accuracy shown in Figs. 2a-c, it is convenient to consider separately several distinct factors which limit precision in GoughReitzel variometers.

\subsection{Resolution in the scaling of records}

The points plotted as deflection amplitudes represent arithmetic means of three independent scalings of the same event. Vertical error bars centred on the points have lengths equal to twice the standard deviations of the means. Standard deviations have also been calculated for individual scalings, and the mean of such values with their extrema are entered in Table 1.

Table 1.

\begin{tabular}{|c|c|c|c|c|}
\hline \multicolumn{2}{|l|}{ All values in $\mathrm{nT}$. } & $H$ sensors & $D$ sensors & $Z$ sensors \\
\hline $\begin{array}{l}\text { Scaling consistency; (Sect. 3.1) Standard } \\
\text { deviations of individual record scal- } \\
\text { ings. }\end{array}$ & $\begin{array}{l}\text { worst case } \\
\text { best case } \\
\text { mean, all cases }\end{array}$ & $\begin{array}{l}0.5 \\
0.04 \\
0.3\end{array}$ & $\begin{array}{l}0.7 \\
0.08 \\
0.1\end{array}$ & $\begin{array}{l}0.5 \\
0.03 \\
0.2\end{array}$ \\
\hline $\begin{array}{l}\text { Calibration consistency; (Sect. 3.2) Std } \\
\text { dvtns of individual calibration de- } \\
\text { flectns. Calibration field changes were } \\
65 \text { for } H, 142 \text { for } D, 71 \text { for } Z \text {. }\end{array}$ & $\begin{array}{l}\text { worst case } \\
\text { best case } \\
\text { mean, all cases }\end{array}$ & $\begin{array}{l}3.7 \\
0.2 \\
0.9\end{array}$ & $\begin{array}{l}1.9 \\
0.5 \\
1.2\end{array}$ & $\begin{array}{l}3.3 \\
0.2 \\
1.2\end{array}$ \\
\hline $\begin{array}{l}\text { Instrument deflection consistency; (Sect. } \\
\text { individual points in Figs. } 2 \mathrm{a}-\mathrm{c} \text { from t1 } \\
\text { fit. }\end{array}$ & $\begin{array}{l}\text { 3) Std dvtns of } \\
\text { ir lines of best }\end{array}$ & $\begin{array}{c}0.5 \\
\text { (Fig. 2b) }\end{array}$ & $\begin{array}{c}1.3 \\
\text { (Fig. 2a) }\end{array}$ & $\begin{array}{c}1.6 \\
\text { (Fig. 2c) }\end{array}$ \\
\hline
\end{tabular}

\subsection{Accuracy of the calibration process}

The calibration process adopted is that in which a thin magnet in an aluminium cube is placed in different orientations on the top face of a variometer. Upon the accuracy and consistency of the calibration deflections thus caused depend all amplitude estimates which follow. The calibration values plotted in Figs. 2a-c result from taking arithmetic means of single scalings of up to eleven different calibration deflections. Horizontal error bars centred on the points have lengths equal to twice the standard deviations of such means. Standard deviations have also been computed for single calibration estimates, and the means of these with their extrema are entered in Table 1. Such standard deviations are greater than can be attributed to scaling, and on the assumption that the calibration procedure has been carried out correctly each time, they imply that the response of the sensors to the calibrating fields is to a degree inconsistent. Such inconsistency cannot be simply due to sensor interaction, instrument 
installation error, or temperature drift. Together with evidence to follow, it will be interpreted in Section 3.5 as slight non-linear behaviour in the mechanical sensor suspensions.

\subsection{Linearity and consistency of response in the $H, D$ and $Z$ sensing systems}

Graphs of amplitudes recorded by a new instrument against amplitudes recorded by the Toolangi observatory have been plotted for a variety of events. Figure 2d, an example, shows that to first order the $H$ sensor of instrument 12 is indeed linear relative to the observatory. Of perhaps more interest, however, the individual instrument 12 scaled values miss the straight line of Fig. $2 \mathrm{~d}$ with a standard deviation of $1.1 \mathrm{nT}$, indicating a lack of precision greater than that which can be due to scaling alone: evidently non-linear or inconsistent response has been detected.

Similar examination of the points plotted in Figs. 2a-c gives estimates of the residuals by which these points miss their respective lines of best fit. Standard deviations for individual points are entered in Table 1 . The value of $1.1 \mathrm{nT}$ from Fig. $2 d$ is of the same order as the estimates from Figs. 2a-c.

\subsection{Interactions between different sensing elements}

In a variometer, let the $H$ sensor be $d_{1} \mathrm{~cm}$ vertically above and $d_{2} \mathrm{~cm}$ to the north of the $Z$ sensor. Let the $H$ and $Z$ sensors be of moments $M_{H}$ and $M_{Z}$ respectively, and let a positive increase in the ambient $H$ field of $\Delta H$ deflect the $H$ sensor through angle $\delta \theta_{H}$. Whereas the moment $M_{H}$ was initially east-west there are now moments $M_{H} \sin \delta \theta_{H}$ in the north direction and $M_{H} \cos \delta \theta_{H}$ eastwest. For small deflections $\sin \delta \theta_{H} \simeq \delta \theta_{H}$ and $\cos \delta \theta_{H} \simeq 1$; thus to first order all that need be considered is the new moment $M_{H} \delta \theta_{H}$ in the horizontal north direction. At the $Z$ sensor, this moment will cause a downwards vertical field $\delta Z_{H}$ of

$$
\delta Z_{H}=-\frac{3 d_{1} d_{2}}{\left(d_{1}^{2}+d_{2}^{2}\right)^{5 / 2}} M_{H} \delta \theta_{H}
$$

which is the spurious $Z$ field caused by the ambient change of $\Delta H$ through the $Z-H$ interaction.

Similarly an ambient change of $\Delta Z$ causes a spurious $H$ field at the $H$ sensor of

$$
\delta H_{Z}=-\frac{3 d_{1} d_{2}}{\left(d_{1}^{2}+d_{2}^{2}\right)^{5 / 2}} M_{Z} \delta \theta_{Z}
$$

For the instruments as operated at Toolangi, values of $\delta \theta_{H}$ ranged from $1.1 \times 10^{-4}$ to $2.6 \times 10^{-4}$ radians per $\mathrm{nT}$, and of $\delta \theta_{z}$ from $0.4 \times 10^{-4}$ to $2.0 \times 10^{-4}$ radians per $\mathrm{nT}$. (Relevant here is a correction to be made to the Gough-Reitzel 
paper, p. 206, where in lines 3 and 5 the $f \theta$ and $2 f \theta$ should be $2 f \theta$ and $4 f \theta$ respectively.) A typical value for both $M_{Z}$ and $M_{H}$ is 90 gauss-cm $\mathrm{cm}^{3}$, and for the present instruments with $d_{1}=25.2 \mathrm{~cm}$ and $d_{2}=4.93 \mathrm{~cm}$, ranges for $\delta Z_{H}$ and $\delta H_{Z}$ are computed to be:

$$
\begin{array}{lll}
\delta Z_{H}=-0.033 & \text { to }-0.078 \mathrm{nT} \text { in } Z \text { per nT in } H, \\
\delta H_{Z}=-0.015 \text { to }-0.060 \mathrm{nT} \text { in } H \text { per nT in } Z .
\end{array}
$$

For Toolangi, the amplitude $\Delta Z$ of a short-period $Z$ disturbance is approximately 0.2 times the amplitude $\Delta H$ of a simultaneously occurring $H$ disturbance, i.e.

$$
\Delta Z \simeq 0.2 \Delta H \text {. }
$$

Thus the spurious $\delta Z_{H}$ effect due to the deflection of an $H$ sensor measuring $\Delta H$ will be in the range:

$$
\delta Z_{H}=-0.033 \Delta H \quad \text { to }-0.078 \Delta H
$$

and the ratio of the $\delta Z_{H}$ interaction to the true $\Delta Z$ occurring is in the range:

$$
\delta Z_{H} / \Delta Z=-0.17 \text { to }-0.39 \text {. }
$$

The $\delta H_{Z}$ interaction, on the other hand, is less important. For Toolangi $\delta H_{Z} / \Delta H$ lies in the range -0.003 to -0.012 . Thus the correction is usually less than $1 \%$, and consequently has not been made to any of the $\Delta H$ data of this paper. The $\delta H_{Z}$ value of $-0.06 \mathrm{nT}$ per $\mathrm{nT}$ above corresponds to an abnormally high $Z$ sensitivity: a value of $-0.02 \mathrm{nT}$ per $\mathrm{nT}$ is more typical of GoughReitzel variometers as they are commonly used. At any observing site let $\Delta Z$ be related to $\Delta H$ by $\Delta Z=A \Delta H$. Then for $\delta H_{Z} / \Delta H$ to approach say $2 \%, A$ has to approach unity, which would only occur for an extremely anomalous station. As a general rule the $\delta H_{Z}$ interaction is evidently negligible. The interactions of the $H$ and $Z$ sensors with the $D$ sensor have also been investigated, and shown negligible.

The agreement of the corrected points in Fig. 2c with the observatory value is some check on the validity of Eq. 1, and the large error of the point which has the greatest correction may be due to limits of the equation being approached.

\subsection{Drift of the sensors}

A major contribution to sensor drift comes from slow relaxation in the suspension fibres, which are re-tensioned at installation. Such drift may be analysed by taking a model which changes towards some equilibrium position with a rate proportional to its deflection from that position. That is

$$
\frac{\mathrm{d} \theta}{\mathrm{d} t}=-\frac{\theta}{\tau}
$$


where $\theta$ is the sensor deflection from ultimate equilibrium, $t$ denotes time, and $\tau$ is a positive constant, a "half-life". The drift rate may also be expressed

$$
\frac{\mathrm{d} \theta}{\mathrm{d} t}=-\frac{\theta_{0}}{\tau} \mathrm{e}^{-t / \tau}
$$

where $\theta_{0}$ is the displacement at zero time. Thus observed between $t_{1}$ and $t_{1}+\delta t$, where $\delta t \ll \tau$, the drift rate will be approximately linear and equal to

$$
\left(\frac{\mathrm{d} \theta}{\mathrm{d} t}\right)_{t=t_{1}}=-\frac{\theta_{0}}{\tau} \mathrm{e}^{-t_{1} / \tau}
$$

At a given time $t_{1}$ after drifting starts, a sensor will show greatest drift if its half-life $\tau$ is equal to $t_{1}$. If several relaxation processes are taking place independently in different parts of a sensor suspension, the short-term drift soon after installation is likely to be different from the long-term drift, and examination of the records after a time $t_{1}$ will tend to show such relaxation processes as are taking place with half-lives near $t_{1}$.

Toolangi variometer records for a particular period of eight days, occurring some five weeks after the instruments had been installed in the ground, have been analysed to determine the drift rates of the instruments in a settled condition. The field differences between two magnetically quiet times were measured for the Toolangi observatory, and for those variometers which recorded all three components at the two times.

Results are presented in Fig. 3. For each component the variometer measurements appear to be normally distributed about the observatory measurement (presumed free of drift), and the most extreme case gives an average drift rate (if it can be approximated as linear) of order $2 \mathrm{nT}$ per day. The mean drift for

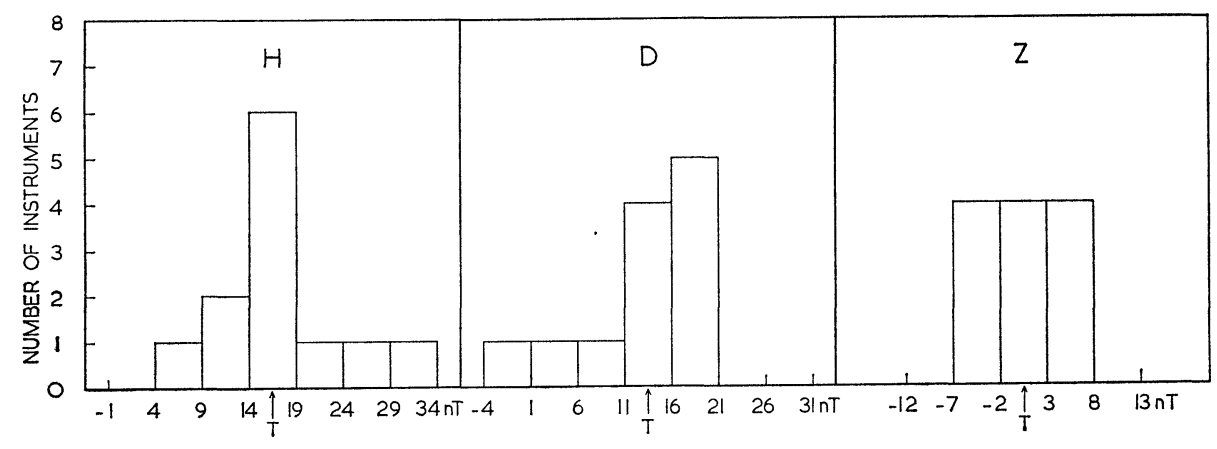

Fig. 3. Histograms showing apparent field changes recorded by twelve instruments from 16 to 24 September, 1973. The "T'" marks on the baseline show changes recorded by the Toolangi observatory. Instrument drift is the difference between apparent field change and the $\mathrm{T}$ mark. 
all sensors, disregarding sign, is of order $1 / 2 \mathrm{nT}$ per day. A great deal depends upon the care and skill with which a suspension fibre is strung.

Five variometer records which showed most drift (three $H$, one $D$ and one $Z$ ), were examined at intermediate times to check whether their drift was indeed simple exponential. This examination showed the drifting to be erratic in some cases, evidently influenced by strong magnetic activity. Such portions of drift curves as did approximate exponential decays indicated half-lives in a range from two months to several days, and initial deflections in a range from fifty to several hundred $\mathrm{nT}$.

The evidence that strong activity initiated new drift may correlate with the slight inconsistency found in the sensor responses, remarked upon in Sections 3.2 and 3.3. One possible mechanism could be that strong deflection of a sensor causes a slight zero-shift in its equilibrium position. Gough and ReITzel (1967) comment that sensors attain fresh equilibrium positions for every reading, as between readings a strong transient field occurs (from the camera solenoid). They reason that the succession of such readings as smooth traces establishes the consistency of sensor response. The Toolangi data indicate that deflections which are more varied than the repetitive transients may in fact cause sensors to adopt less consistent equilibrium positions.

\subsection{Accuracy in instrument construction and installation}

Running the instruments simultaneously in the same place tests together the uniformity of construction and the uniformity of installation. The scatter of points in Figs. 2a-d is accounted for by the inconsistent response evident in calibrations. No claim can be made for evidence of installation inaccuracy, unless the standard deviations in the lowest line of Table 1 are not accounted for by those in the line above. For $Z$ the possibility remains that a standard deviation of order $1 \mathrm{nT}$ has been observed in the scatter of Fig. 2c, perhaps best attributed to installation inaccuracy.

\subsection{Accuracy of the timing process}

Good data for checking timing occurred during 18 August to 11 September. Time checks were recorded at the start and finish of this period, and a sharp event occurring on 29 August was determined from nine records to have commenced at $09 \mathrm{hr} 36 \mathrm{~m} 39 \mathrm{~s}$, this mean time having a standard deviation of $3 \mathrm{~s}$, and the standard deviation of an individual time estimate being $7 \mathrm{~s}$. As the onset of the sharp event was interpolated from data recorded at $30 \mathrm{~s}$ intervals, this check detected no timing errors in the instruments.

The percentage error in the timing of data is thus likely to be much less than the percentage error in the amplitude of data. When analysing records in 
the frequency domain, there is therefore an advantage in using parameters of amplitude and phase, which keep clearly distinct errors due to two measuring systems which are clearly distinct.

\section{Conclusion}

For the data presented, it is evident that while single scalings of a record can be made with a standard deviation of order $0.2 \mathrm{nT}$, the record itself is likely to be in error with a standard deviation of $1.5 \mathrm{nT}$. The causes of this error are inconsistency and non-linearity in the sensor responses during both recording and calibration, and possibly inaccuracy in the orientation and levelling of the instruments in the ground. With scalings from four different calibration deflections, a standard deviation of $1 \%$ is probably a safe expectation in calibration estimates.

The simple modification of interchanging the positions of the $H$ and $D$ sensors in the instruments should obviate sensor interactions. Other improvements may not be possible without complicating a design specifically intended to be simple and economical.

Construction of the instruments was made possible by the generosity of Professor D.I. Gough, who lent a variometer to be the pattern of a new set. J.M. Kennedy designed the electronic circuitry, and we thank N. Riebeek, M. Laybutt and J. Kras for assistance. At Toolangi we were grateful for the help of Mr. and Mrs. R. Biggs, and for use of the facilities of the Bureau of Mineral Resources. We also much appreciated the hospitality of Bob and Pam Pritchard.

\section{REFERENCES}

Gough, D.I. and J.S. Reitzel, A portable three-component magnetic variometer, J. Geomag. Geoelectr., 19, 203-215, 1967.

Serson, P.H., Instrumentation for induction studies on land, Phys. Earth Planet. Inter., 7, 313322, 1973. 